\title{
Measurements of the Radar Cross Section of a nano-drone at K-band
}

\author{
Alessio Balleri \\ Centre for Electronic Warfare, Information and Cyber \\ Cranfield University, Defence Academy of the UK \\ Shrivenham, SN6 8LA, UK \\ a.balleri@cranfield.ac.uk
}

\begin{abstract}
Nano-drones, are insect-like sized drones with a threat capability of intrusion to provide intelligence and potentially violate secure establishments and public privacy rights. They are an existing technology which is becoming increasingly more available, portable, affordable and easy to operate. As such, they represent a plausible defence and security threat.

In this paper, a setup is presented that is used to measure the 3dimensional Radar Cross Section (RCS) of a nano-drone for three elevation planes with a 5-degree resolution step in azimuth and elevation. The results presented in this paper are unique because there has been very little work, if any, in the existing literature attempting to measure the RCS of such small drones. They are also key to inform further work investigating the development of nano-drone detection radar systems as well as nano-drone detection and classification signal processing solutions.

Index Terms-Radar Cross Section (RCS), nano-drones, picodrones, drone signatures, drone classification, drone recognition, drone detection
\end{abstract}

\section{INTRODUCTION}

Nano-drones are unmanned aerial systems of a size comparable to that of insects that can potentially be used to perform stealthy surveillance or to gather intelligence, even within enclosed locations and buildings. According to the classification of drones offered in [1], nano-drones are those unmanned flying systems with dimensions ranging between 15 $\mathrm{cm}$ and $2.5 \mathrm{~cm}$ and weighing between $50 \mathrm{~g}$ and $3 \mathrm{~g}$. There also exists other weight based classification systems of drones, such as those proposed in [2] and [3], and they all have very similar weight ranges for each class.

Most of the available research literature on detection and classification of drones focuses on mini-drones and micro-drones, i.e. drones weighing a few kilograms and with dimensions comparable to those of birds.

A helicopter and a quadcopter UAV with rotor lengths of 70 $\mathrm{cm}$ and $20 \mathrm{~cm}$, respectively, were measured in [4] using a Xband Continuous Wave (CW) radar. The micro-Doppler signatures were extracted using the Short Time Fourier Transform (STFT) and used to measure the maximum Doppler velocity shift and to determine the rotor size of the drones. In [5], [6] and [7] it was shown that the use of long window STFT produces frequency lines in the spectrogram that repeat up to the maximum Doppler frequency of the rotor tip. The separation between each line is a factor of the number of propeller blades and the rotation rate of the propeller and are called HElicopter Rotor Modulation (HERM) lines. An example of the signature of a DJI Phantom 3 quadcopter micro-UAV in flight can be seen in [5]. A new method using HERM lines to extract the rotation rate of the propellers of different drones, based on a log harmonic summation algorithm which is commonly used in audio processing, was proposed in [6].

Research into the RCS of drones demonstrated that the material of the blade as well as the drone overall size and shape can significantly affect the micro-Doppler target returns. The use of carbon, metal and plastic rotor blades was investigated on a DJI drone platform and results were compared in [8]. Measurements of a DJI Phantom Vision 2 flying a specific flight path were compared to similar sized birds (such as the hooded vulture, the eurasian eagle owl and the barn owl) flying the same flight path in [9]. A similar experiment was conducted using K-band and W-band radars in [10]. The use of radar polarimetry was investigated in [11] as a potential method to distinguish UAVs from birds. Additional results on RCS measurements of mini and micro drones can be found in [12], [13] and [14].

As most of the existing radar literature investigating drone detection and classification relates to the case of larger drones, conventional anti-drone radar systems have been designed and optimised to detect and classify bigger targets at long distance rather than nano-targets.

Measurements of a nano-UAV were conducted in [15] where a $35 \mathrm{GHz} \mathrm{CW}$ radar designed for 3D imaging was used to conduct a micro-Doppler analysis of a variety of UAVs, including mini, micro and nano-UAVs. Previous research aiming at designing and prototyping a flexible K-band FMCW radar to detect and collect micro-Doppler signatures of nano drones was presented in [16] and [17]. Results showed that the micro-Doppler signatures of an Arcade Pico-Drone could be successfully gathered in a laboratory environment, at a short range, with little transmitted average power. However, this research did not attempt to measure and calibrate the RCS of the drone. Some other relevant comparable literature investigates radar detection and classification of insects with wingspans in the range of the centimetres, that is similar to the size of the propeller blades found in nano-UAVs. In [18], for example, a W-band radar and a S-band radar were used to measure the micro-Doppler signature of different 
species of moths with wingspans in the range of $30-100 \mathrm{~mm}$. Using the W-band radar, the authors were able to detect and measure the micro-Doppler signature of all the moths under test allowing an estimation of the wing beat frequencies with good precision.

The detection of nano drones is an emerging problem that needs immediate address. In this paper, a measurement setup available at Cranfield University at the Defence Academy of the UK is presented that allows measurements of the RCS of small targets in 3D. The setup is used to measure the RCS of a nano-drone (the Arcade pico-drone) and results are reported and analysed. Understanding and measuring the RCS of nano drones are fundamental key steps towards developing detection algorithms. Moreover, this study will help develop signal processing techniques for classification.

\section{EXPERIMENTAL SETUP}

The measurement setup consists of a LinearX precision turntable, an MS46322A Anritsu Vector Network Analyser (VNA), a PC and a wooden arch with a diameter of $2 \mathrm{~m}$ that hosts two identical standard $(5.5 \times 4 \mathrm{~cm})$ horn antennas. The arch is designed so that the antennas can be manually moved along the arch with an elevation resolution step of 5 degrees. A photo of the setup is shown in Fig. 1. The turntable carries a styrofoam stand and is positioned, with a laser alignment system (as shown in Fig. 2) so that the position of the target under test coincides with the centre of the arch. The turntable is capable of rotating with an angular resolution of 0.1 degrees and can be fully controlled remotely with a set of pre-defined commands which are sent through a serial cable from the PC. The antennas are arranged so that they transmit and receive in vertical polarisation (VV). Rotating the turntable allows measurements of the target response from different azimuthal aspect angles, whilst moving the antenna positions on the arch allows measurements from different elevation planes with a step of 5 degrees. The Anritsu VNA can operate in the range of frequencies from $40 \mathrm{MHz}$ up to $40 \mathrm{GHz}$ and is operated so that, at each aspect angle, it measures the amplitude and phase of the impulse response of the target over a pre-defined bandwidth $B$ with a maximum of up to 16,001 frequency points.

The turntable and the VNA are connected to the PC with a serial cable and an ethernet cable, respectively, and are fully controlled and synchronised with LabView. For each elevation angle, the horizontal turtable is rotated over 360 degrees to collect the target signature as a function of the aspect angle in azimuth. The Labview scripts were developed so to ensure that the VNA collects the target frequency response before triggering the stepped rotation of the turntable. A userdefined waiting time is also included between rotation steps in order to ensure the setup is completely stationary during each measurement.

\section{RESULTS}

The results presented in this paper were obtained with measurements collected in K-band, from $23 \mathrm{GHz}$ to $25 \mathrm{GHz}$,

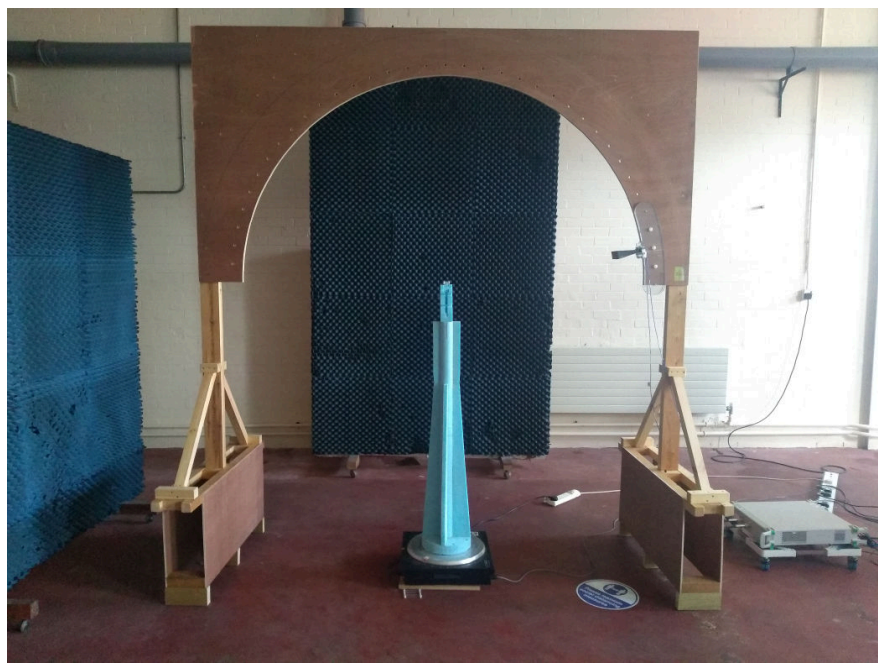

Fig. 1. Photo of the setup with horns pointing the drone from an aspect angle of 10 degrees in elevation.
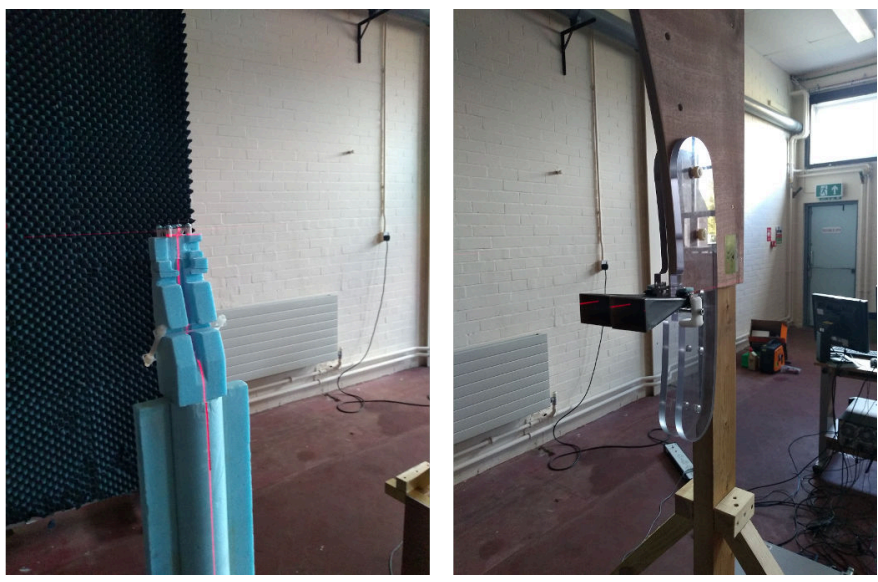

Fig. 2. Photo of the aligned setup showing the laser vertical and horizontal lines on the target and horns.

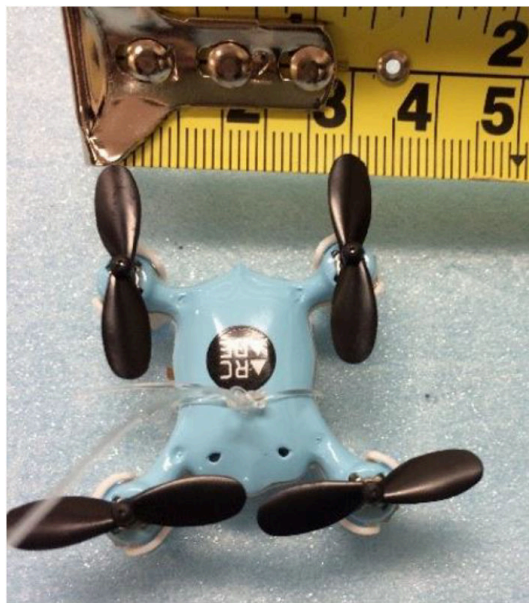

Fig. 3. Photo of the Arcade Pico-drone. 
TABLE I

PARAMETERS OF THE CALIBRATING SPHERES. THE EXPECTED THEORETICAL RCS IS CALCULATED UNDER THE ASSUMPTION THE SPHERES ARE PERFECT Electric CONDUCTORS (PEC).

\begin{tabular}{|c|c|c|c|}
\hline $\begin{array}{c}\text { Diameter } \\
(\mathrm{mm})\end{array}$ & $\begin{array}{c}\text { Theoretical RCS } \\
(\mathrm{dB})\end{array}$ & $\begin{array}{c}\text { Measured Response } \\
(\mathrm{dB})\end{array}$ & $\begin{array}{c}\text { Cal } \\
(\mathrm{dB})\end{array}$ \\
\hline 30 & -31.62 & 120.96 & -152.58 \\
\hline 20 & -34.78 & 117.31 & -152.09 \\
\hline 10 & -39.92 & 112.45 & -152.37 \\
\hline 8 & -42.87 & 109.22 & -152.09 \\
\hline
\end{tabular}

over 8,001 frequency points. Some Radar Absorbent Material (RAM) was positioned around the setup to mitigate the effects of unwanted reflections from the surrounding open laboratory area. The target compressed time-responses were obtained in post-processing using a sub-band $B$ of $1 \mathrm{GHz}$ around a central frequency of $24 \mathrm{GHz}$, i.e. with a range resolution of $15 \mathrm{~cm}$. Calibration was carried out by measuring the response of four different spheres of different diameters, as reported in Table I. Multiple off-the-shelf metallic spheres were used to provide four independent calibration parameters, and therefore increase calibration fidelity, because of the uncertain morphological precision, material composition and density of the spheres.

Fig. 4 shows the range-compressed response of the four sphere. Results show a very clean $\sin (x) / x$ response around the target position with peaks at the correct target range of $1 \mathrm{~m}$, as well as the expected $-13 \mathrm{~dB}$ sidelobes and range resolution of $15 \mathrm{~cm}$. Differences between the peak response levels between spheres and the corresponding theoretical expected RCS values calculated as in [19] were used to generate the four independent calibration offsets reported in Table I. Results show a very good agreement between the four calibration parameters. The signature of the $3 \mathrm{~cm}$ diameter sphere was measured, as a function of aspect angle with a 5 degree resolution in azimuth, for 0,5 and 10 degrees in elevation to establish the robustness of the experimental setup as a function of angle. Two background measurements were taken for each azimuth and elevation pair and subtracted from the sphere returns to investigate the dependency of the result on any background cancellation errors.

Fig. 5 shows the uncalibrated response of the sphere as a function of angle for the two independent background cancellation measurements. Results show that the sphere returns are all within $1.5 \mathrm{~dB}$ and this also gives an indication of the expected accuracy of the measurement setup for such small targets at this range of frequencies.

An Arcade Pico drone, whose micro-Doppler signatures were collected in [16], was used as the target under test for the RCS measurements. A photo of the Arcade Pico drone with an indication of the drone size is shown in Fig. $3 .{ }^{1}$ The farfield $R=50 \mathrm{~cm}$ was calculated using the relationship

$$
R=\frac{2 D^{2}}{\lambda}
$$

\footnotetext{
${ }^{1}$ Note that, despite the name used by the supplier, the Arcade Pico drone falls under the nano drone category according to the classification in [1]
}

where $D$ is the largest target/antenna dimension $(5.5 \mathrm{~cm})$ and $\lambda$ is the wavelength at $24 \mathrm{GHz}$.

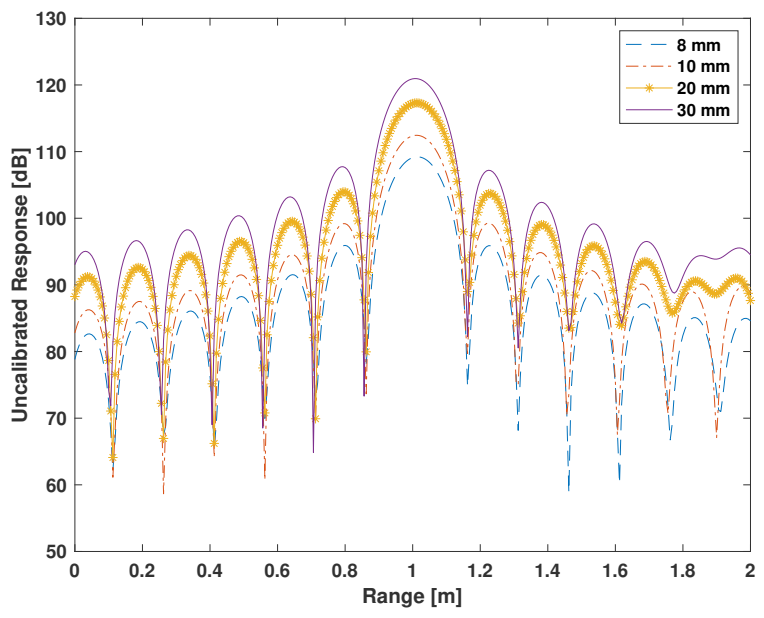

Fig. 4. Response of the four calibrating spheres as a function of range for $B=1 \mathrm{GHz}$.

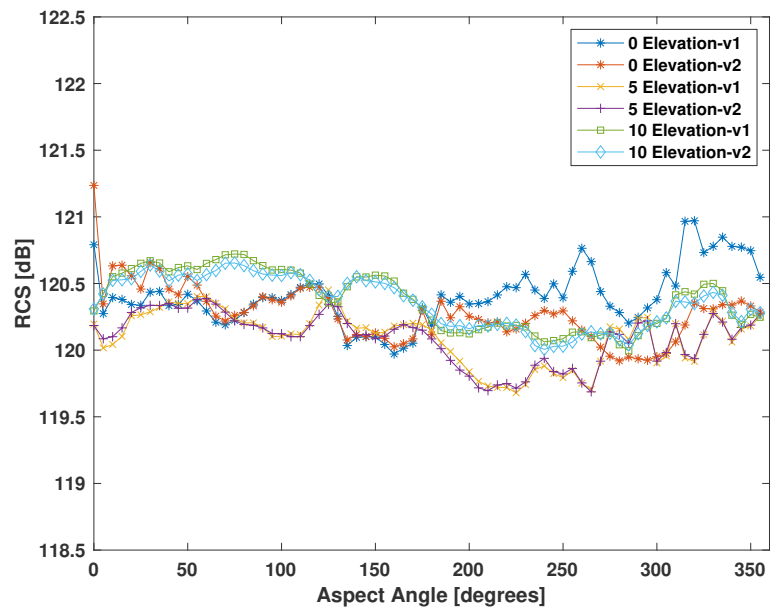

Fig. 5. Measured returns of the $3 \mathrm{~cm}$-diameter sphere as a function of aspect angle and elevation. Two results are presented for each angle to show the level of robustness of the results with respect to background cancellation.

Fig. 6 and Fig. 7 show the calibrated response of the Arcade pico-drone (calibrated using the values reported in Table I) as a function of range and azimuth, for an elevation angle of 0 degrees, and its relative cut at the target range, respectively. Results show that the RCS of the drone presents peaks at around $-25 \mathrm{~dB}$ as well as significant RCS variations with aspect angle spanning RCS values down to about $-55 \mathrm{~dB}$. Background cancellation results at 0 degrees in elevation showed that taking the difference between two background measurements resulted in returns around $-70 \mathrm{~dB}$ for azimuth angles below 200 degrees and higher values, with peaks reaching $-51 \mathrm{~dB}$, for azimuth angles higher than 200 degrees. For these reasons, the measurements of the drone RCS that 
fall below $-40 \mathrm{~dB}$ (i.e. less than $10 \mathrm{~dB}$ than the background cancellation performance) at angles greater than 200 degrees might be less accurate than the measurements taken from lower aspect angles. Similar results are shown relative to an elevation angle of 5 degrees in Fig. 8 and Fig. 9, and for 10 degrees in Fig. 10 and Fig. 11. At both 5 and 10 degrees in elevation, background cancellation performance were in the order of -70 $\mathrm{dB}$ at all azimuth angles indicating a much better accuracy of the results for RCS levels around $-50 \mathrm{~dB}$. The setup presented in this paper was initially constructed to measure targets with a much higher RCS than that of nano-drones. Background cancellation performance at this range of frequencies and for such small targets can be further improved by the construction of a higher performing styrofoam stand and this is something that will be further investigated in future work.

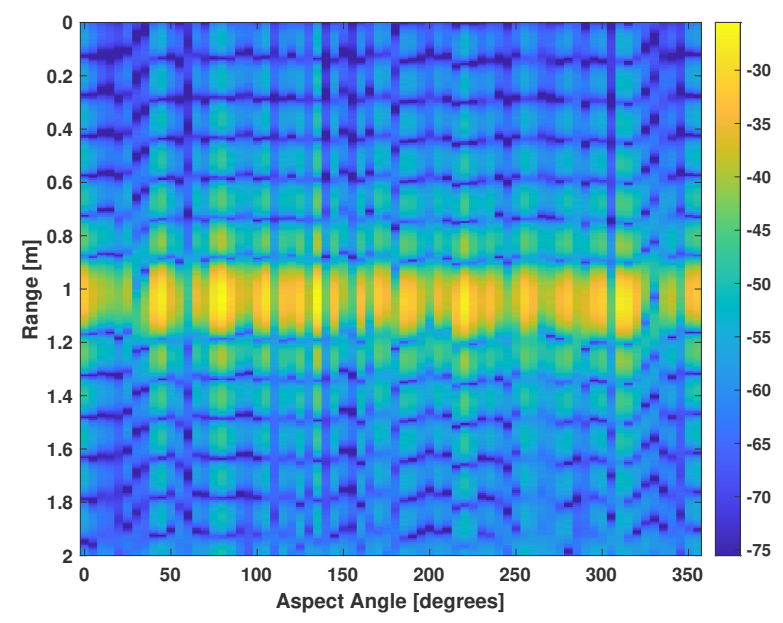

Fig. 6. Calibrated target response as a function of range and azimuth for an elevation angle of 0 degrees. The colorbar is in $\mathrm{dB}$.

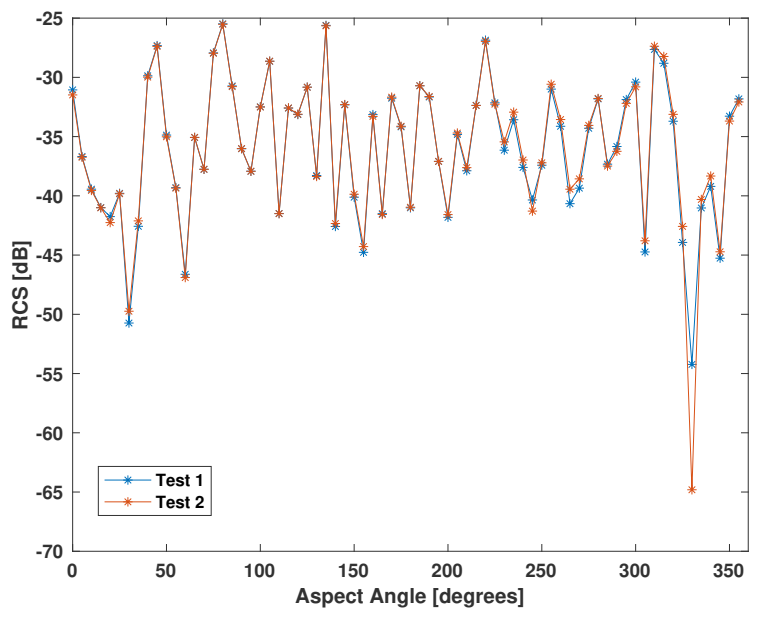

Fig. 7. Calibrated target RCS as a function of aspect angle for an elevation angle of 0 degrees. Results are given for the subtraction of two independent background measurements.

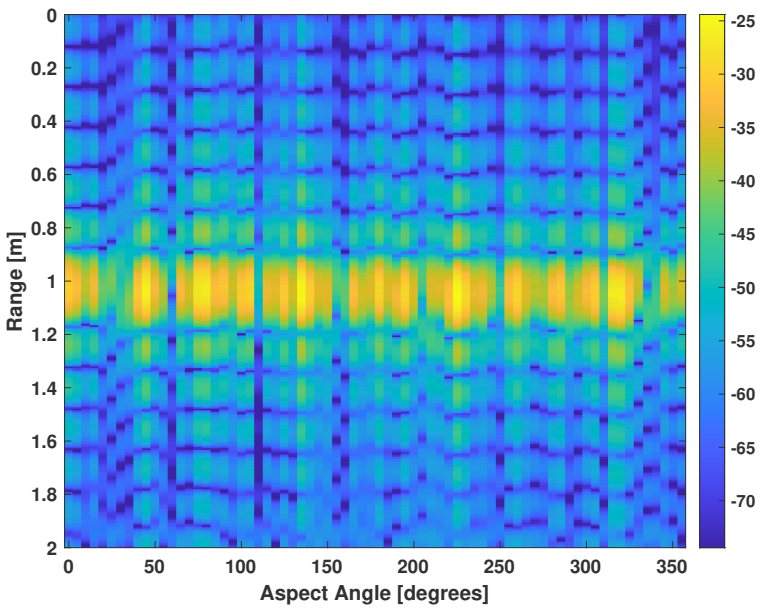

Fig. 8. Calibrated target response as a function of range and azimuth for an elevation angle of 5 degrees. The colorbar is in $\mathrm{dB}$.

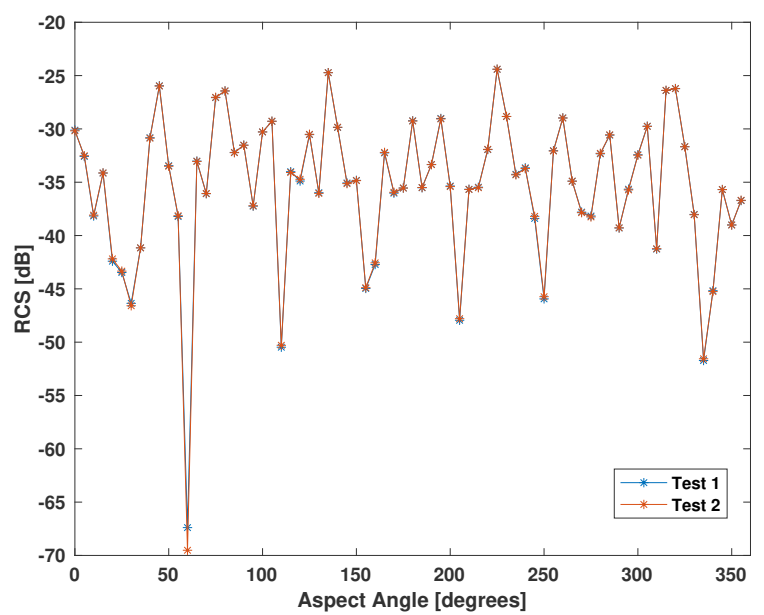

Fig. 9. Calibrated target RCS as a function of aspect angle for an elevation angle of 5 degrees. Results are given for the subtraction of two independent background measurements.

\section{CONCLUSIONS}

The RCS of an Arcade pico-drone has been measured, for three different elevation planes, using a 3D measurement setup that allowed data collection as a function of elevation angle (with a 5-degree resolution) and azimuth angle (with a 5degree resolution). Results have shown the Arcade pico-drone presents peak RCS values just over $-25 \mathrm{~dB}$ (i.e. $0.0032 \mathrm{~m}^{2}$ ) as well as significant variations as a function of aspect angle (down to less than about $-50 \mathrm{~dB}$ ). The results presented in this paper are timely and fundamental as there has been very little prior work in the existing literature on detection and classification of nano-drones and very little, if any, attempting to measure their RCS. The results presented in this paper are to inform further studies on detection of nano-drones, particularly to aid the analysis of the performance of detection algorithms 


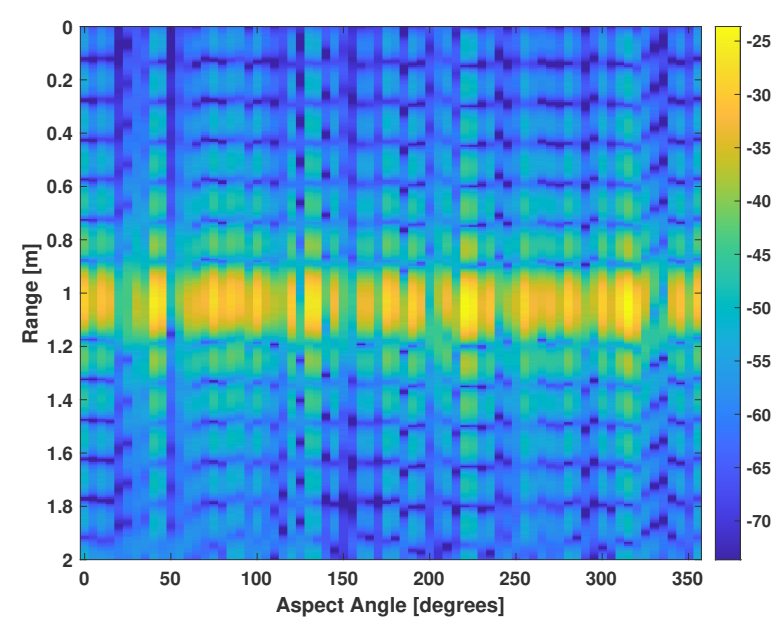

Fig. 10. Calibrated target response as a function of range and azimuth for an elevation angle of 10 degrees. The colorbar is in $\mathrm{dB}$.

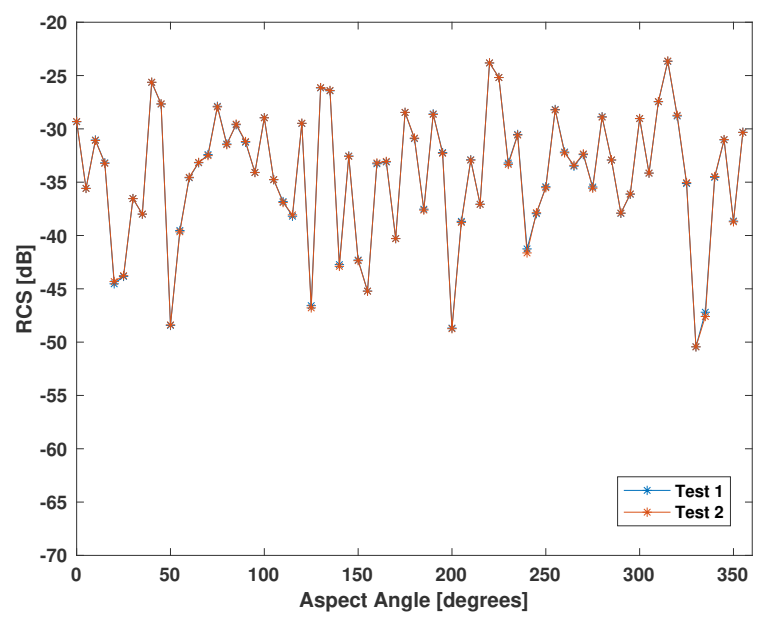

Fig. 11. Calibrated target RCS as a function of aspect angle for an elevation angle of 10 degrees. Results are given for the subtraction of two independent background measurements.

in different and challenging deployment scenarios (e.g. [20]).

\section{REFERENCES}

[1] M. Hassanalian and A. Abdelkefi, "Classifications, applications, and design challenges of drones: A review," Progress in Aerospace Sciences, vol. 91, pp. 99-131, 2017.

[2] R. Weibel and R. J. Hansman, Safety Considerations for Operation of Different Classes of UAVs in the NAS, 2004.

[3] M. Arjomandi, "Classification of unmanned aerial vehicles," Ph.D. dissertation, University of Adelaide, 2007.

[4] J. J. M. de Wit, R. I. A. Harmanny, and G. Prémel-Cabic, "Micro-doppler analysis of small uavs," in 2012 9th European Radar Conference, 2012, pp. $210-213$.

[5] S. Rahman and D. A. Robertson, "Coherent $24 \mathrm{GHz}$ FMCW radar system for micro-Doppler studies," in Radar Sensor Technology XXII, K. I. Ranney and A. Doerry, Eds., vol. 10633, International Society for Optics and Photonics. SPIE, 2018, pp. $154-162$.

[6] A. Huang, P. Sévigny, B. Balaji, and S. Rajan, "Fundamental frequency estimation of herm lines of drones," in 2020 IEEE International Radar Conference (RADAR). IEEE, 2020, pp. 1013-1018.
[7] S. Rahman and D. A. Robertson, "Millimeter-wave micro-Doppler measurements of small UAVs," in Radar Sensor Technology XXI, K. I. Ranney and A. Doerry, Eds., vol. 10188, International Society for Optics and Photonics. SPIE, 2017, pp. $307-315$.

[8] M. Ritchie, F. Fioranelli, H. Griffiths, and B. Torvik, "Micro-drone rcs analysis," in 2015 IEEE Radar Conference, 2015, pp. 452-456.

[9] — "Monostatic and bistatic radar measurements of birds and microdrone," in 2016 IEEE Radar Conference (RadarConf), 2016, pp. 1-5.

[10] S. Rahman and D. A. Robertson, "Radar micro-Doppler signatures of drones and birds at K-band and W-band," in Scientific Reports, vol. 8, 2018 , pp. $1-11$.

[11] B. Torvik, K. E. Olsen, and H. Griffiths, "Classification of birds and uavs based on radar polarimetry," IEEE Geoscience and Remote Sensing Letters, vol. 13, no. 9, pp. 1305-1309, 2016.

[12] S. Pisa, E. Piuzzi, E. Pittella, P. Lombardo, A. Genovese, D. Bloisi, D. Nardi, P. d'Atanasio, and A. Zambotti, "Numerical and experimental evaluation of the radar cross section of a drone," in 2018 15th European Radar Conference (EuRAD), 2018, pp. 309-312.

[13] M. Ezuma, M. Funderburk, and I. Guvenc, "Compact-range rcs measurements and modeling of small drones at $15 \mathrm{ghz}$ and $25 \mathrm{ghz}$," in 2020 IEEE Radio and Wireless Symposium (RWS), 2020, pp. 313-316.

[14] V. Semkin, J. Haarla, T. Pairon, C. Slezak, S. Rangan, V. Viikari, and C. Oestges, "Analyzing radar cross section signatures of diverse drone models at mmwave frequencies," IEEE Access, vol. 8, pp. 48958 48969, 2020.

[15] L. Fuhrmann, O. Biallawons, J. Klare, R. Panhuber, R. Klenke, and J. Ender, "Micro-doppler analysis and classification of uavs at ka band," in 2017 18th International Radar Symposium (IRS), 2017, pp. 1-9.

[16] S. Zulkifli and A. Balleri, "Design and development of k-band fmcw radar for nano-drone detection," in 2020 IEEE Radar Conference (RadarConf20), 2020, pp. 1-5.

[17] — "FMCW radar prototype development for detection and classification of nano-targets," in 2020 IEEE International Radar Conference (RADAR), 2020, pp. 738-743.

[18] R. Wang, C. Hu, X. Fu, T. Long, and T. Zeng, "Micro-doppler measurement of insect wing-beat frequencies with w-band coherent radar," Scientific reports, vol. 7, no. 1, pp. 1-8, 2017.

[19] B. R. Mahafza, Radar systems analysis and design using MATLAB. Chapman and Hall/CRC, 2013.

[20] F. Gini, M. Greco, and A. Farina, "Clairvoyant and adaptive signal detection in non-gaussian clutter: a data-dependent threshold interpretation," IEEE Transactions on Signal Processing, vol. 47, no. 6, pp. 1522-1531, 1999. 